\title{
Star Formation Around the Youngest Supernova Remnants in the Large Magellanic Cloud: Implications for Type Ia Supernova Progenitors
}

\author{
Carles Badenes*, Jason Harris ${ }^{\dagger}$, Dennis Zaritsky** and José Luis Prieto ${ }^{\ddagger}$ \\ ${ }^{*}$ Department of Astrophysical Sciences, Princeton University, Peyton Hall, Ivy Lane, Princeton NJ \\ 08544-1001; Chandra Fellow \\ ${ }^{\dagger}$ National Optical Astronomy Observatory, 950 North Cherry Ave., Tucson, AZ 85719 \\ ${ }^{*}$ Steward Observatory, 933 North Cherry Ave., Tucson, AZ 85721 \\ ${ }^{\ddagger}$ Department of Astronomy, Ohio State University, McPherson Laboratory, 140 W. 18th Avenue.
}

Columbus, $\mathrm{OH} 43210$

\begin{abstract}
We use the star formation history map of the Large Magellanic Cloud recently published by Harris \& Zaritsky to study the sites of the youngest Type Ia supernova remnants. We find that most Type Ia remnants are associated with old, metal-poor stellar populations, with little or no recent star formation. These include SNR 0509-67.5 which is known to have been originated by an extremely bright SN 1991T-like event, and yet is located very far away from any star forming regions. It is very unlikely that this bright Type Ia SN had a young stellar progenitor. The Type Ia remnant SNR N103B, however, is associated with vigorous star formation activity in the last 100 $\mathrm{Myr}$, and might have had a relatively younger and more massive progenitor.
\end{abstract}

Keywords: supernova remnants — supernovae:general — galaxies: stellar content — galaxies: individual: Large Magellanic Cloud

PACS: $26.30 . \mathrm{k}$ 95.85.Nv 97.60.Bw

\section{A NEW WAY TO CONSTRAIN THE PROPERTIES OF SUPERNOVA PROGENITORS}

After decades of efforts, the fundamental properties of Type Ia supernova (SN) progenitors still remain obscure (see [1] and references therein). The reason is simple: although hundreds of new Type Ia SNe are discovered each year, their host galaxies are too far away to either detect the the faint progenitors in pre-explosion images [2] or study the stellar populations around the explosions with enough detail. Most of the times, all that can be learned from the study of the hosts is an average age and metallicity for the entire galaxy [3].

Focusing on Local Group galaxies has the advantage that the stellar populations can be resolved and studied in exquisite detail. Waiting for SNe to explode in these galaxies is obviously not practical, but there is no need for that. Recent advances in our understanding of the X-ray emission from Type Ia supernova remnants (SNRs) [4, 5] have made it possible to determine the fundamental properties of Type Ia SNe by studying their SNRs (see [6] for an example). In some cases it has been possible to verify the results obtained from the SNR with the spectroscopy of the light echoes associated with the parent SN itself (e.g. [7, 8] for the case of SNR 0509-67.5). We 

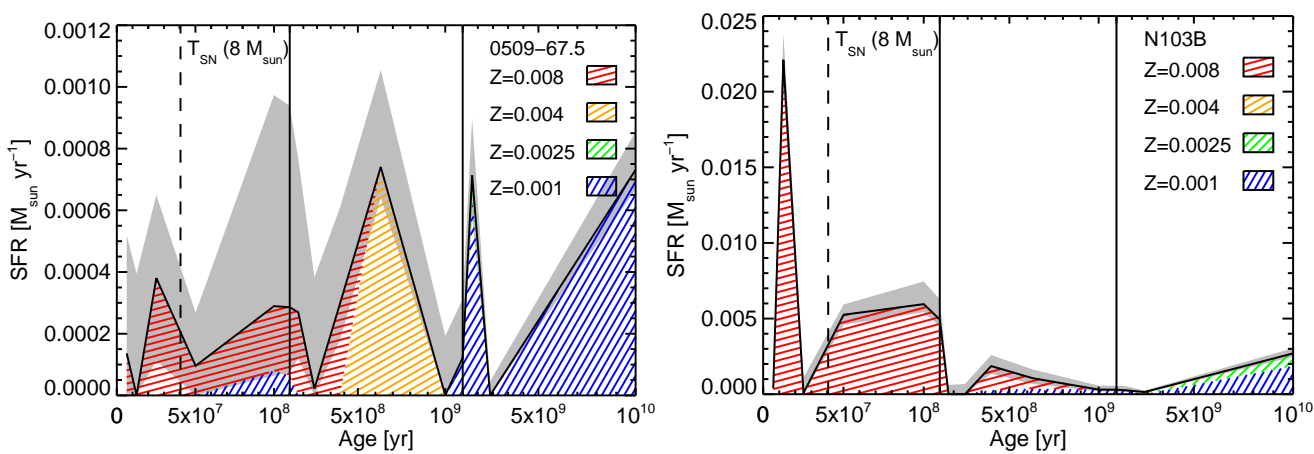

FIGURE 1. Local SFHs around the Type Ia SNRs 0509-67.5 (left panel) and N103B (right panel), broken down in four metallicity bins. The grey area corresponds to the error bar on the total SFH.

take advantage of these techniques to study the stellar star formation history (SFH) map of the LMC published by Harris and Zaritsky [9], which was produced by applying the StarFISH code [10] to the data from the Magellanic Clouds Photometric Survey [11].

Our results will be presented in a forthcoming publication (Badenes et al. 2009, in preparation). In Figure 1 we show the SFHs associated with two of the four Type Ia SNRs in our study. From both its X-ray spectrum [8] and the SN light echo [7], SNR 0509-67.5 is known to have been originated by an exceptionally bright, SN 1991-T like Type Ia SN. However, the stellar population around it is very old (average age 7.9 Gyr) and metal-poor (average $Z$ 0.0014). The SNR is located in a region of the LMC called the Northwestern Void, very far away from any sites of recent star formation [9]. This is in contrast to the prevalent view that bright Type Ia SNe come from younger and possibly more massive progenitors Gallagher et al. [12], Scannapieco and Bildsten [13]. The situation for SNR N103B is very different. The SFH is dominated by a vigorous episode of recent star formation, with one extended peak between 50 and $100 \mathrm{Myr}$ ago and a more brief outburst 12 Myr ago. This Type Ia SNR might have had a more massive and younger progenitor than SNR 0509-67.5.

\section{REFERENCES}

1. D. Maoz, MNRAS 384, 267-277 (2008).

2. D. Maoz, and F. Mannucci, MNRAS 388, 421-428 (2008).

3. J. L. Prieto, K. Z. Stanek, and J. F. Beacom, ApJ 673, 999-1008 (2008).

4. C. Badenes, E. Bravo, K. J. Borkowski, and I. Domínguez, ApJ 593, 358 (2003).

5. C. Badenes, K. J. Borkowski, and E. Bravo, ApJ 624, 198-212 (2005).

6. C. Badenes, K. J. Borkowski, J. P. Hughes, U. Hwang, and E. Bravo, ApJ 645, 1373-1391 (2006).

7. A. Rest et al., ApJ 680, 1137-1148 (2008).

8. C. Badenes, J. P. Hughes, G. Cassam-Chenaï, and E. Bravo, ApJ 680, 1149-1157 (2008).

9. J. Harris, and D. Zaritsky, ApJ (2008), submitted.

10. J. Harris, and D. Zaritsky, ApJS 136, 25-40 (2001).

11. D. Zaritsky, J. Harris, I. B. Thompson, and E. K. Grebel, AJ 128, 1606-1614 (2004).

12. J. S. Gallagher et al., ApJ 634, 210-226 (2005).

13. E. Scannapieco, and L. Bildsten, ApJ 629, L85-L88 (2005). 